\title{
Construction of a Novel Risk Model Based on the Random Forest Algorithm to Distinguish Pancreatic Cancers with Different Prognoses and Immune Microenvironment Features
}

\section{Yalan Lei}

Fudan University Shanghai Cancer Center

\section{Rong Tang}

Fudan University Shanghai Cancer Center Jin Xu

Fudan University Shanghai Cancer Center

Bo Zhang

Fudan University Shanghai Cancer Center Jiang Liu

Fudan University Shanghai Cancer Center

Chen Liang

Fudan University Shanghai Cancer Center

Qingcai Meng

Fudan University Shanghai Cancer Center

Jie Hua

Fudan University Shanghai Cancer Center

Xianjun Yu

Fudan University Shanghai Cancer Center

\section{Wei Wang}

Fudan University Shanghai Cancer Center

Si Shi ( $\sim$ shisi@fudanpci.org )

Fudan University Shanghai Cancer Center

\section{Research}

Keywords: immune-related IncRNA, pancreatic cancer, random survival forest, immune infiltration, risk model

Posted Date: May 5th, 2021 
DOl: https://doi.org/10.21203/rs.3.rs-454599/v1

License: (c) (1) This work is licensed under a Creative Commons Attribution 4.0 International License. Read Full License

Version of Record: A version of this preprint was published at Bioengineered on January 1st, 2021. See the published version at https://doi.org/10.1080/21655979.2021.1951527. 


\section{Abstract}

Background: Immune-related long noncoding RNAs (irlncRNAs) have been demonstrated to be actively involved in the regulation of immune status. With this study, we aimed to establish a risk model of irlncRNAs and further investigate the roles of irlncRNAs in prognosis prediction and the immune landscape in pancreatic cancer.

Methods: The transcriptome profiles and clinical information of pancreatic cancer patients were retrieved from The Cancer Genome Atlas (TCGA). Immune-related genes (irgenes) downloaded from ImmPort were used to screen the IncRNAs by correlation analysis $(R>0.5, p<0.001)$. Random survival forest (RSF) and survival tree analysis showed that 9 irlncRNAs were highly correlated with overall survival (OS) according to the variable importance (VIMP) and the minimal depth. Then, Cox regression was used to establish a risk model with 3 irlncRNAs, which was evaluated by Kaplan-Meier analysis. In addition, we performed Cox regression analysis to establish the clinical prognostic model, which showed that the risk score was the only independent prognostic factor $(p<0.001)$. A nomogram was drawn to visualize the clinical features. Wilcoxon signed-rank test and analysis of the correlations between the risk score and immune cells was applied to explore the potential irlncRNAs related to immune status.

Results: A total of 176 samples were randomly divided into a training set $(n=123)$ and a test set $(n=53)$. A total of 1903 irlncRNAs were identified after Pearson correlation analysis between irgenes and IncRNAs. A total of 9 irlncRNAs were identified in the survival tree analysis, and 3 irlncRNAs (LINC00462, LINC01887, RP11-706C16.8) were used to establish a risk model. The areas under curve (AUC) of the receiver operating characteristic (ROC) for 36 months, 30 months, 24 months, 18 months, 12 months and 6 months were $0.778,0.774,0.751,0.753,0.780$ and 0.756 , respectively, with a concordance index (C-index) of 0.696 . In the clinical prognostic model, the C-index increased from 0.599 to 0.682 after combining the risk scores. Furthermore, a high risk was associated with increased infiltration of CD4+ T cells, M0 macrophages and M1 macrophages.

Conclusions: We established a novel three-irlncRNA risk model. The clinical evaluation showed its robustness in predicting the prognosis and immune landscape of pancreatic cancer.

\section{Backgrounds}

Pancreatic cancer is a highly malignant tumor with a 5 -year survival rate of less than $10 \%$, and it is the seventh leading cause of death in developed countries [1]. The short overall survival (OS) highlights the need for an accurate staging system to predict the prognosis, and the modification of the $8^{\text {th }}$ edition of the American Joint Committee on Cancer (AJCC) staging system contributes to this [2]. Surgical excision is the only way to achieve a complete cure, and chemotherapy and neoadjuvant treatment play an important role in pancreatic cancer therapy. Although immunotherapy has made great progress in several tumors, mono- or combined immune checkpoint inhibitors show limited effects in pancreatic cancer, which may partially be due to the reduced infiltration of immune cells, poor immunogenic immune 
microenvironment, and abundant mesenchymal fibroblasts blocking drug delivery [3, 4]. An understanding of the immune microenvironment of pancreatic cancer is urgently needed to promote its clinical application.

Long noncoding RNAs (IncRNAs) account for over 80\% of RNAs, and their transcripts are over 200 nucleotides in length; IncRNAs do not translate into proteins. LncRNAs interfere with proteins, RNA and DNA to participate in many biological regulation processes, including transcriptome modulation and gene modification [5]. In addition, recent studies have delineated the mechanisms of IncRNAs that are actively involved in tumor biology, such as H19, PVT1, NEAT1 and HISLA, which were disclosed to be associated with tumorigenesis, epithelial mesenchymal transition, metastasis, chemoresistance, immune evasion and metabolic reprogramming [6-8].

Immune-related IncRNAs (irlncRNAs) have recently been studied in several cancers. Wang et al [9] identified 4 irlncRNAs to establish a risk model for lung adenocarcinoma, while 8 irlncRNAs were used to construct a prognostic model for melanoma [10]. Moreover, irlncRNAs have also utilized in glioblastoma, head and neck squamous cell carcinoma and bladder cancer [11-13]. These models demonstrate the potential clinical significance of irlncRNAs and provide novel insights for establishing a clinical prognostic model.

In this article, we retrieved the transcriptome profiling data and clinical information of patients in The Cancer Genome Atlas pancreatic cancer dataset (TCGA-PAAD). After correlation analysis of IncRNAs with immune-related genes (irgenes), irlncRNAs were identified. The irlncRNAs underwent differential expression analysis, least absolute shrinkage and selection operator (LASSO) regression analysis and Cox regression analysis to establish a novel and robust risk model. Then, the risk model was evaluated in the validation cohort. The irlncRNA signatures were analyzed with other clinical characteristics, and we established a clinical prognostic model. Furthermore, irlncRNA signatures were demonstrated to be associated with immune cell infiltration in pancreatic cancer.

\section{Results}

\section{Identification of immune-related long noncoding RNAs (irlncRNAs)}

The process flow of this study is shown in Figure 1. First, we obtained the transcriptome profile from highthroughput sequencing (HTseq) count data $\left(n_{\text {patient }}=176, n_{\text {normal }}=4\right)$ and the clinical information of patients from The Cancer Genome Atlas pancreatic cancer dataset (TCGA-PAAD). Second, we retrieved the gene transfer files (GTFs) from Ensembl to annotate the IncRNAs from the expression matrix. Third, immune-related genes (irgenes) were downloaded from the ImmPort database. Pearson correlation analysis between the IncRNAs and irgenes was completed, and 1903 irlncRNAs were identified $(R>0.5$, $\mathrm{p}<0.001)$ (Table S1).

\section{Establishment of risk model by random survival forest (RSF) analysis}


RSF was applied constructed to determine the irlncRNAs that are of most significance to the OS of pancreatic patients. The samples were randomly divided into a training set $(n=123)$ and a test set $(n=53)$ at a ratio of 7:3. In the survival tree analysis, the 9 most significant variables (CTC-529P8.1, RP11706C16.8, LINC01493, LINC01887, LINC00462, LINC01510, LINC02205, RP11-1082L8.2 and RP11402N8.1) were selected according to the variable importance (VIMP) and the minimal depth. The subsequent Cox regression analysis identified 3 irlncRNAs (LINC00462, LINC01887, RP11-706C16.8), with a coefficient index and risk score for each sample in the training set calculated. To validate this model, the receiver operating characteristic (ROC) curves were drawn, and the areas under the curves (AUCs) for 36 months, 30 months, 24 months, 18 months, 12 months and 6 months were $0.778,0.774,0.751,0.753$, 0.780 and 0.756 , respectively (Figure 2$)$. In addition, the C-index for this risk model was $0.696(p<0.001)$.

\section{Clinical evaluation of the risk model}

Instead of the median risk score value, the best cutoff value calculated by X-Tile software (https://medicine.yale.edu/lab/rimm/research/software/) was utilized to divide the samples into highrisk and low-risk groups in the training set and validation set. It was 1.44 in training set and 1.40 in test set. To clinically evaluate the risk model, several analytical methods were applied. First, Kaplan-Meier analysis showed that the OS of patients in the high-risk group was significantly lower than that of patients in the low-risk group ( $p<0.001$ ). In the high-risk group, the 5 -year OS rate was $5.66 \%$, and the $95 \%$ confidence interval $(\mathrm{Cl})$ was 0.88 to 361 ; the 5 -year OS rate was $30.9 \%$, and the $95 \% \mathrm{Cl}$ was 18.28 to 52.4 in the low-risk group. In the test set, Kaplan-Meier analysis showed a significant difference in OS between the two groups $(p<0.001)$ (Figure 3$)$.

\section{The risk model is an independent prognostic factor for pancreatic cancer}

To construct a more accurate clinical prognostic risk model, the AUC of each ROC for every clinical characteristic and the risk score was calculated; the AUCs of the risk score, age, sex, T stage, N stage, $\mathrm{M}$ stage and stage were $0.778,0.654,0.548,0.564,0.686,0.462$ and 0.557 , respectively, with the risk score AUC being the only one above 0.7 . After univariate Cox regression analysis, the risk score $(p<0.001), N$ $(p=0.006)$ and $T(p=0.034)$ were selected for multivariate Cox regression analysis, which revealed that the risk score $(p<0.001)$ was the only independent prognostic factor for pancreatic cancer (Table 1). Moreover, the addition of the risk score to the clinical model can raise the C-index from 0.599 to 0.682 , indicating that it greatly contributes to prognosis prediction. A nomogram and the related calibration plots were established to visualize the specific method, calculate the risk scores and show the ability of the model to predict OS at 6 months, 12 months and 36 months (Figure 4).

\section{Exploring the correlation between immune cell infiltration and the risk model}

The irlncRNAs identified by correlation analysis of irgenes and IncRNAs at the beginning of this study may exert an influence on immune status, including immune cell infiltration. We employed the Tumor IMmune Estimation Resource (TIMER), CIBERSORT, QUANTISEQ, MCPcounter and EPIC resources to delineate the immune cell infiltration of the training set (Table S2). Wilcoxon signed-rank analysis 
between the risk score and immune cell infiltration revealed that high risk was associated with greater infiltration of cancer-associated fibroblasts, follicular helper T cells, CD4+ T cells, M0 macrophages and M1 macrophages, while low risk was correlated with greater infiltration of B cells and M2 macrophages. Pearson correlation analysis showed the correlation between the risk score and the infiltration of 3 types of immune cells ( $M 0$ macrophages, CD4+ T cells and M1 macrophages) $(p<0.1)$ (Table S3). The correlation results were expressed in a lollipop graph. However, the Wilcoxon signed-rank test comparing risk and immune checkpoint inhibitor (ICI) biomarkers, including CTLA4, LAG3, ID01, PDCD1 and ICOS, showed no significant association, which is reflective of the poor effect of ICls in clinical trials (Figure 5).

\section{Discussion}

In this research, we established a risk model and clinical prognostic model with 3 irlncRNAs and explored the correlation between the irlncRNA signature and immune cell infiltration. First, correlation analysis of IncRNAs and irgenes was performed to obtain irlncRNAs. Second, random survival forest, survival tree decision and Cox regression analyses were applied to establish the risk model by irlncRNAs. In addition, we calculated the AUC of the time-dependent ROC curve to validate its practical significance. Third, to confirm its clinical significance, Kaplan-Meier analysis, chi-square test, Wilcoxon signed-rank test, and Cox regression analysis were utilized and confirmed that irlncRNA signature was the only independent prognostic factor among clinical characteristics. Finally, we explored the relationship between the irlncRNA signature and immune cell infiltration, which revealed that specific immune cells differentially infiltrated tissues from the high- and low-risk groups, shedding light on the immune microenvironment of pancreatic cancer. The risk model established from irlncRNAs in this research has high predictive value. After combining the risk score into the clinical prognostic model, the C-index increased from 0.599 to 0.682 , indicating that it significantly contributes to clinical prognosis efficacy among pancreatic cancer patients. Furthermore, the irlncRNA signature can predict the immune landscape, including immune cell infiltration in tumor tissue, thus providing insights for immunotherapy.

IncRNAs have been widely applied in predicting the prognosis of different cancers in the past few months. A total of 3 IncRNAs were identified to establish a risk model in pancreatic cancer, and they were discovered to be correlated with epithelial metastasis transition (EMT) [14]. A risk model composed of 5 IncRNAs was associated with cancer-related and drug response pathways in pancreatic ductal adenocarcinoma [15]. Several other studies have also taken advantage of IncRNAs to construct prognostic models [16-18]. These studies indicate that IncRNAs play a role in tumor biological functions, including chemotherapy resistance, tumorigenesis and EMT. Our research focused on the potential immune functions of IncRNAs and conducted integrated clinical evaluation of the risk model. To improve the efficacy and accuracy, we performed validation in another cohort and applied multiple analyses and databases to provide insights into exploring the tumor immune microenvironment. Wei et al. [19] disclosed the potential biological functions of irlncRNAs in pancreatic tumors, indicating that tumor purity is negatively associated with the infiltration of fibroblasts, myeloid dendritic cells and monocytes. This finding paved the way for further research on irlncRNAs and their potential regulatory mechanisms in pancreatic cancer. 
IncRNAs have special localization features and functional mechanisms; for example, they can assemble with proteins, RNA and DNA to participate in various biological processes. Previous studies have revealed that IncRNAs are correlated with tumorigenesis, tumor progression, metabolism and immune status; thus, they have gradually become drug targets [20]. For example, TARID can methylate TCG21 to promote tumorigenesis, and CCAT1- $L$ is involved in tumor progression, metastasis and drug resistance [21, 22]. Of note, IncRNAs may play a role in regulating the tumor immune microenvironment by activating immune cells, promoting immune evasion or other mechanisms [23]. NKILA is a typical IncRNA that can regulate the apoptosis sensitivity of different types of T cells, and the silencing of NKILA can improve immunotherapy effects in breast cancer $[7,12]$. The irlncRNAs identified in our research included LINC00462, LINC01884, and RP11-706C16.8, and some of them have been previously studied. For example, LINC00462 participates in the miR-666/TGFBR1-TGFBR2/SMAD2/3 or AKT signaling pathways to promote tumor invasion and progression in pancreatic cancer and hepatic cancer [24-26]. Further research on the mechanisms of the identified irlncRNAs may reveal their potential regulatory function in the tumor microenvironment.

Despite the fact that immunotherapy has made great progress in tumor treatment, it shows limited effects and many side effects in pancreatic cancer. Some clinical trials on immunotherapy in pancreatic cancer, such as ICls applied alone or combined with chemotherapy (NCT02558894, NCT02331251); immune vaccines (NCT00084383); CD40 antibodies (NCT00711191); and mesenchymal target therapy (NCT02734160), are currently under way [27-31]. In this research, we conducted Pearson correlation analysis of the irlncRNA signature and common biomarkers of ICls. However, there was no significant correlation, which is consistent with the limited immunotherapy effect in pancreatic cancer [27, 32]. Pancreatic cancer is usually regarded as poorly immunogenic and is accompanied by fewer mutated antigens recognized by patient $T$ cells than lung cancer and melanoma [33]. However, this research showed that high risk was associated with increased infiltration of cancer-associated fibroblasts, CD4 + T cells, M0 macrophages and M1 macrophages, while low risk was correlated with M2 macrophage infiltration, indicating that macrophage differentiation may be correlated with malignancies and may be a potential target in immunotherapy.

There are limitations to this bioinformatic research. First, the TCGA-PAAD database lacks information on immunotherapy, which limits further evaluation of the clinical prognostic model in predicting the response to immunotherapy. Second, the limited number of samples may influence the risk model established with irlncRNAs. Third, this research lacks outside validation for the nonoverlapping irlncRNAs in the Gene Expression Omnibus (GEO) database. To improve the accuracy, we applied the random survival forest model and completed integrated analysis to confirm the robustness of the risk model.

In conclusion, we established a novel and robust risk model with 3 irlncRNAs and a 3-year survival AUC of 0.778. The risk model was identified as the only prognostic factor in the clinical evaluation and can delineate the immune landscape of pancreatic cancer, which has potential clinical value.

\section{Materials And Methods}




\section{Retrieval of the transcriptome data and identification of immune-related IncRNAs (irlncRNAs)}

We obtained the transcriptome profile data (high-throughput sequencing (HTseq) counts) and clinical information of patients $\left(n_{\text {patient }}=176, n_{\text {normal }}=4\right)$ from The Cancer Genome Atlas pancreatic cancer dataset (TCGA-PAAD). Then, we downloaded the gene transfer files (GTFs) from Ensemble (http://asia.ensembl.org) to annotate the transcriptome profiles and extract the IncRNA expression profiles. In addition, immune-related genes (irgenes) were downloaded from the ImmPort database (http://www.immport.org), and a total of 2438 genes were obtained. After a Pearson correlation analysis of IncRNAs and irgenes, 1903 irlncRNAs were identified ( $r>0.5, p<0.001)$. In this step, the Hmisc package was employed.

\section{Establishment of the risk model by random survival forest (RSF) analysis}

The RSF model was applied to determine the irlncRNAs that were of significance to the OS and survival status according to variable importance (VIMP) and the minimal depth. Survival tree analysis was constructed according to the variables selected from the previous procedure (CTC-529P8.1, RP11706C16.8, LINC01493, LINC01887, LINC00462, LINC01510, LINC02205, RP11-1082L8.2, RP11-402N8.1), with 2000 trees and the log-rank splitting rule. After deleting the variables with extremely low expression, the risk model was established by multivariable Cox regression analysis. A total of 3 variables (LINC00462, LINC01887, RP11-706C16.8) were selected by Cox regression analysis and were used to predict the risk score for each sample in the training set. The forest map of the 3 irlncRNAs was drawn. In addition, the areas under the receiver operating characteristic (ROC) curves (AUCs) for 36 months, 30 months, 24 months, 18 months, 12 months and 6 months as well as the concordance index (C-index) for the risk model were calculated. The randomForestSRC, glmnet, survival, surviminer, ggplot2, forestplot, survcopm, and prodlim packages were used in this procedure.

\section{Clinical validation of the risk model}

According to X-Tile software (https://medicine.yale.edu/lab/rimm/research/software/), the best cutoff value for the risk score was determined to be 1.44 in the training set and 1.40 in the test set. After dividing the samples into high-risk and low-risk groups in the training set and test set separately, Kaplan-Meier analysis was conducted to analyze the difference in OS in the high- and low-risk groups. Furthermore, we drew survival plots and survival curves to visualize the difference between the two groups. The survivaIROC, plotROC, ggplot2, survival and survminer packages were applied in this step.

\section{Establishment of the clinical prognostic model}

To evaluate the clinical significance of the irlncRNAs in the signature, we further conducted several analyses, including Cox regression analysis and Pearson correlation analysis. The AUC for each clinical characteristic and the risk score were calculated to determine the potential prognostic factors. Univariate Cox regression and multivariate Cox regression analysis were applied to construct the clinical prognostic model. A nomogram was constructed and a calibration plot was drawn to show the results of Cox 
analysis. The C-index of the clinical prognostic model increased from 0.599 to 0.682 after taking the risk score into consideration. The dplyr, ggolot2, ggpubr, survival, survminer, rms, and survcomp packages were used in this step.

\section{Exploring the correlation between the irlncRNA signature and immune cell infiltration}

Correlation analysis of IncRNAs and irgenes was conducted initially. Therefore, we further explored the correlation between the irlncRNA signature and immune cell infiltration by employing the Tumor IMmune Estimation Resource (TIMER), CIBERSORT, XCELL, QUANTISEQ, MCPcounter and EPIC databases. The Wilcoxon signed-rank test showed the significantly infiltrated immune cells $(p<0.1)$, and Pearson correlation analysis revealed the correlation index between immune cells and the risk score. In addition, we performed the Wilcoxon signed-rank test to assess the association between immune checkpoint inhibitor (ICl) biomarkers and risk. The Hmisc, ggplot2 and ggrepel packages were used here.

\section{Abbreviations}

Irgenes: immune-related genes; irlncRNA: immune-related long non-coding RNA; AUC: Area Under Curve; ROC: Receiver Operating Characteristic;

\section{Declarations}

\section{Ethics approval and consent to participate}

Not applicable

\section{Consent for publication}

Not applicable

\section{Availability of data and materials}

The datasets supporting the conclusions of this article are available in the the cancer genome atlas (TCGA), immPort and TIMER database.

\section{Competing Interests}

The authors declare that they have no competing interests.

\section{Funding}

This study was funded by the National Natural Science Foundation of China (No. 81802352, 81772555 and 81902428), the National Science Foundation for Distinguished Young Scholars of China (No. 81625016), the Shanghai Sailing Program (No. 19YF1409400 and 20YF1409000), the Shanghai RisingStar Program (No. 20QA1402100), the Shanghai Anticancer Association Young Eagle Program (No. 
SACA-CY19A06), the Clinical and Scientific Innovation Project of Shanghai Hospital Development Center (No. SHDC12018109 and SHDC12019109) and the Scientific Innovation Project of Shanghai Education Committee (No. 2019-01-07-00-07-E00057).

\section{Authors' contributions}

(I) Conception and design: LYL and TR; (II) Administrative support: LYL; (III)

Provision of study materials or patients: LYL and TR; (IV) Collection and

assembly of data: LYL and WW; (V) Data analysis and interpretation: LYL, TR and XJ; (VI) Manuscript writing: all authors. All authors read and approved the final manuscript.

\section{Acknowledgements}

We thank American Journal Experts (AJE) for their assistance with language editing, and we also would like to acknowledge the TCGA, immPort database.

\section{References}

1. Rawla P, Sunkara T, Gaduputi V,et al. Epidemiology of Pancreatic Cancer: Global Trends, Etiology and Risk Factors. World J Oncol. 2019;10(1):10-27.

2. Shi S, Hua J, Liang C, Meng Q, Liang D, Xu J, et al. Proposed Modification of the 8th Edition of the AJCC Staging System for Pancreatic Ductal Adenocarcinoma. Ann Surg. 2019;269(5):944-50.

3. Ryan DP, Hong TS, Bardeesy N, et al. Pancreatic adenocarcinoma. N Engl J Med. 2014. doi:10.1056/NEJMc1412266.

4. Feig C, et al. The pancreas cancer microenvironment. Clin Cancer Res. 2012;18(16):4266-76.

5. Dahariya S, et al. Long non-coding RNA: Classification, biogenesis and functions in blood cells. Mol Immunol. 2019;112:82-92.

6. Chen F, et al. Extracellular vesicle-packaged HIF-1alpha-stabilizing IncRNA from tumour-associated macrophages regulates aerobic glycolysis of breast cancer cells. Nat Cell Biol. 2019;21(4):498-510.

7. Huang $D$, et al. NKILA IncRNA promotes tumor immune evasion by sensitizing $T$ cells to activationinduced cell death. Nat Immunol. 2018;19(10):1112-25.

8. Ping G, Xiong W, Zhang L, Li Y, Zhang Y, Zhao Y, et al. Silencing long noncoding RNA PVT1 inhibits tumorigenesis and cisplatin resistance of colorectal cancer. Am J Transl Res. 2018;10(1):138-49.

9. Wang J, et al. Identification and Validation of a Novel Immune-Related Four-IncRNA Signature for Lung Adenocarcinoma. Front Genet. 2021. doi:10.3389/fgene.2021.639254.

10. Wang Y, et al. A prognostic model for melanoma patients on the basis of immune-related IncRNAs. Aging. 2021. doi:10.18632/aging.202730. 
11. Li X, et al. A Five Immune-Related IncRNA Signature as a Prognostic Target for Glioblastoma. Front Mol Biosci. 2021;8:632837.

12. Chen $Y$, et al. An immune-related seven-IncRNA signature for head and neck squamous cell carcinoma. Cancer Med. 2021. doi:10.1002/cam4.3756.

13. Zhang $L$, et al. Identification of Immune-Related IncRNA Signature to Predict Prognosis and Immunotherapeutic Efficiency in Bladder Cancer. Front Oncol. 2020;10:542140.

14. Li M, et al. A Novel and Robust Long Noncoding RNA Panel to Predict the Prognosis of Pancreatic Cancer. DNA Cell Biol. 2020;39(7):1282-9.

15. Zhou $\mathrm{CH}$, et al. A Long Non-coding RNA Signature to Improve Prognostic Prediction of Pancreatic Ductal Adenocarcinoma. Frontiers in Oncology; in press.

16. Wu B, et al. Novel three-IncRNA signature predicts survival in patients with pancreatic cancer. Oncol Rep. 2018;40(6):3427-37.

17. Song JK, et al. Five key IncRNAs considered as prognostic targets for predicting pancreatic ductal adenocarcinoma. J Cell Biochem. 2018;119(6):4559-69.

18. Shi $X$, et al. Three-IncRNA signature is a potential prognostic biomarker for pancreatic adenocarcinoma. Oncotarget. 2018;9(36):24248-59.

19. Wei $\mathrm{CM}$, et al. Bioinformatics profiling utilized a nine immune-related long noncoding RNA signature as a prognostic target for pancreatic cancer. J Cell Biochem. 2019;120(9):14916-27.

20. Chen Y, et al. Long non-coding RNAs: From disease code to drug role. Acta Pharm Sin B. 2021;11(2):340-54.

21. Arab K, et al. GADD45A binds R-loops and recruits TET1 to CpG island promoters. Nat Genet. 2019;51(2):217-23.

22. Isoda T, et al. Non-coding Transcription Instructs Chromatin Folding and Compartmentalization to Dictate Enhancer-Promoter Communication and T Cell Fate. Cell. 2017;171(1):103-19.

23. Statello L, et al. Gene regulation by long non-coding RNAs and its biological functions. Nat Rev Mol Cell Biol. 2021;22(2):96-118.

24. Zhou B, et al. Linc00462 promotes pancreatic cancer invasiveness through the miR-665/TGFBR1TGFBR2/SMAD2/3 pathway. Cell Death Dis. 2018;9(6):706.

25. Gong J, et al. Long noncoding RNA linc00462 promotes hepatocellular carcinoma progression. Biomed Pharmacother. 2017;93:40-7.

26. Wang R, Yan Y, Li C. LINC00462 is involved in high glucose-induced apoptosis of renal tubular epithelial cells via AKT pathway. Cell Biol Int. 2019. doi:10.1002/cbin.1123.

27. Ferris RL, et al. Durvalumab with or without tremelimumab in patients with recurrent or metastatic head and neck squamous cell carcinoma: EAGLE, a randomized, open-label phase III study. Ann Oncol. 2020;31(7):942-50.

28. Weiss GJ, et al. A phase lb study of pembrolizumab plus chemotherapy in patients with advanced cancer (PembroPlus). Br J Cancer. 2017;117(1):33-40. 
29. Lutz E, et al. A lethally irradiated allogeneic granulocyte-macrophage colony stimulating factorsecreting tumor vaccine for pancreatic adenocarcinoma. A Phase II trial of safety, efficacy, and immune activation. Ann Surg. 2011;253(2):328-35.

30. Beatty GL, et al. A phase I study of an agonist CD40 monoclonal antibody (CP-870,893) in combination with gemcitabine in patients with advanced pancreatic ductal adenocarcinoma. Clin Cancer Res. 2013;19(22):6286-95.

31. Melisi $D$, et al. Galunisertib plus gemcitabine vs. gemcitabine for first-line treatment of patients with unresectable pancreatic cancer. Br J Cancer. 2018;119(10):1208-14.

32. Brahmer JR, et al. Safety and activity of anti-PD-L1 antibody in patients with advanced cancer. $\mathrm{N}$ Engl J Med. 2012;366(26):2455-65.

33. Moncada R, et al. Integrating microarray-based spatial transcriptomics and single-cell RNA-seq reveals tissue architecture in pancreatic ductal adenocarcinomas. Nat Biotechnol. 2020;38(3):33342.

\section{Tables}

Table 1. The results of univariate and multivariate Cox regression analysis for the clinical prognostic model.

\begin{tabular}{lllllllll}
\hline \multirow{2}{*}{ Items } & \multicolumn{1}{c}{ Univariate Cox regression } & \multicolumn{5}{c}{ Multivariate Cox regression } & \multicolumn{2}{c}{ C-index } \\
\cline { 2 - 7 } & $\mathrm{HR}$ & $95 \% \mathrm{CI}$ & $\mathrm{p}$ & $\mathrm{HR}$ & $95 \% \mathrm{CI}$ & $\mathrm{P}$ & \\
\hline Risk score* & 1.441 & {$[1.250,1.663]$} & $<0.001$ & 1.384 & {$[1.195,1.601]$} & $<0.001$ & 0.682 \\
$\mathrm{~N}^{*}$ & 1.997 & {$[1.217,3.275]$} & 0.006 & 1.794 & {$[1.017,3.164]$} & 0.043 & \\
$\mathrm{~T}^{*}$ & 1.829 & {$[1.048,3.191]$} & 0.034 & 1.855 & {$[0.977,3.525]$} & 0.059 & \\
Stage & 1.319 & {$[0.829,2.097]$} & 0.242 & - & - & - & - & - \\
$\mathrm{M}$ & 0.8561 & {$[0.667,1.099]$} & 0.222 & - & - & - & - & - \\
Age & 1.022 & {$[0.996,1.048]$} & 0.102 & - & - & - & - & - \\
Sex & 1.417 & {$[0.865,2.322]$} & 0.166 & - & - & - & - \\
\hline
\end{tabular}

\section{Figures}




\section{Transcriptome data from TCGA-PAAD}

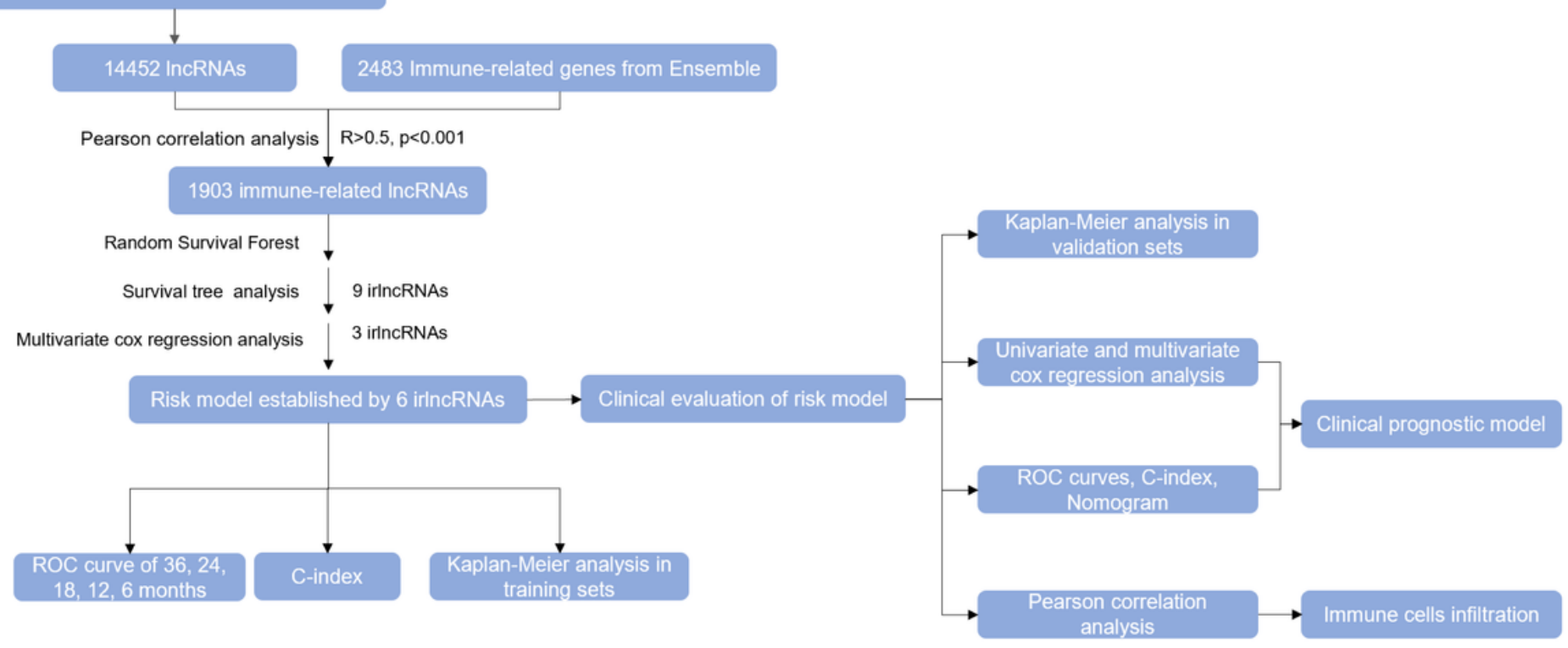

\section{Figure 1}

The process flow to establish the immune-related IncRNAs risk model and its clinical evaluation. 


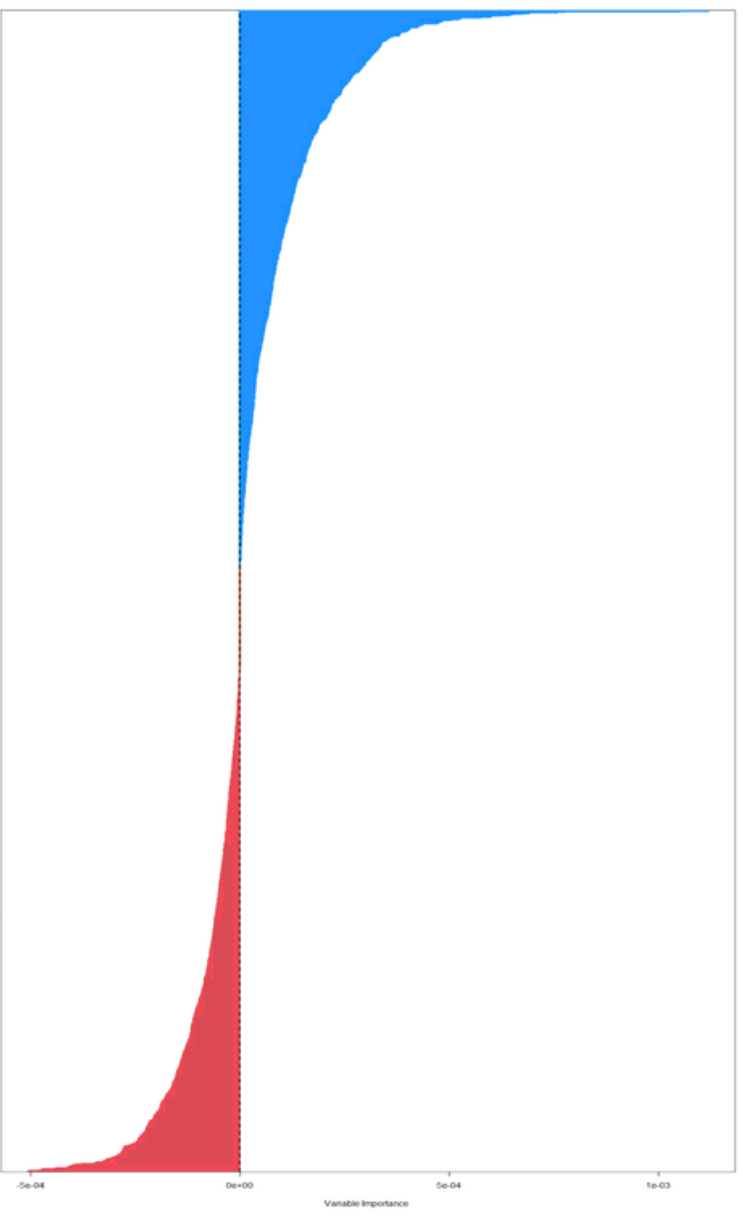

(A)

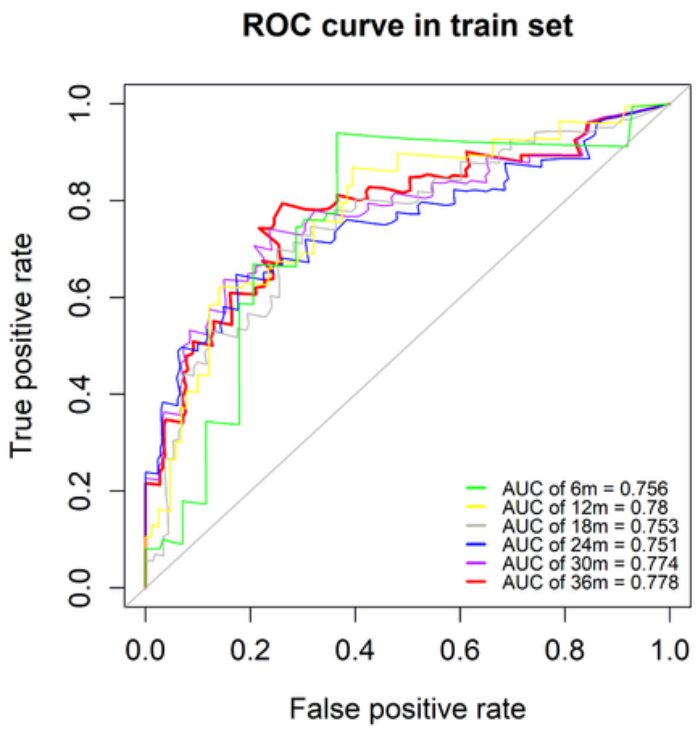

Multivariate cox regression analysis in train set

(C)

ROC curve of clinical characteristics

$\begin{array}{lllll}\text { IncRNA } & \text { HR } & \text { p } & \text { lower } 95 \% \mathrm{Cl} & \text { upper } 95 \% \mathrm{Cl}\end{array}$

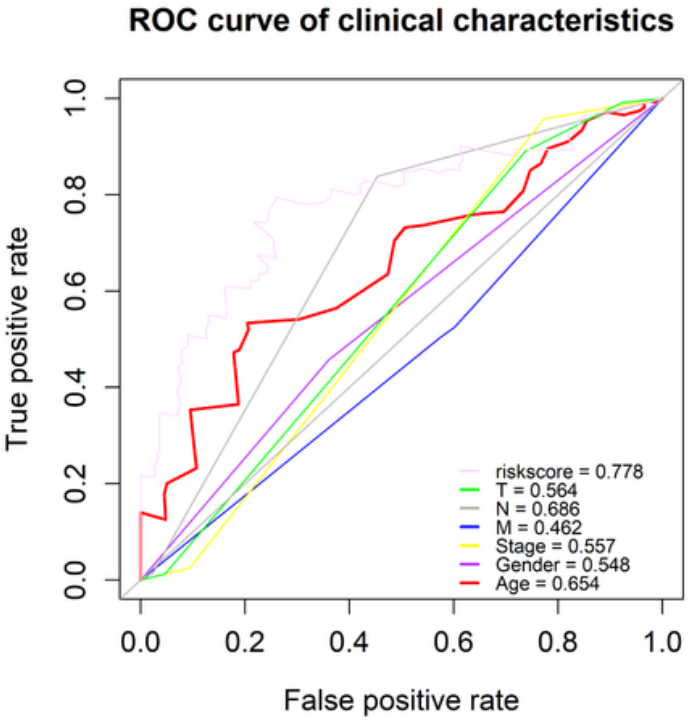

$\begin{array}{lllll}\text { RP11-706C16.8 } & 1.3 & 0.001 & 1.11 & 1.53\end{array}$

\begin{tabular}{lcccc|c}
\hline LINC00462 & 1.13 & 0.093 & 0.98 & 1.3 \\
\hline LINC01887 & 1.14 & 0.02 & 1.02 & 1.28 \\
\hline RP11-706C16.8 & 1.3 & 0.001 & 1.11 & 1.53 & $\square$ \\
\hline
\end{tabular}

(D)

\section{Figure 2}

The establishment of risk model. (A) The important variables selected with the random survival forest model. (B) The forest map of multivariate Cox regression results. (C) The ROC curve of the risk model for survival at 36 months, 30 months, 24 months, 18 months, 12 months and 6 months. (D) The ROC curve of clinical characteristics 

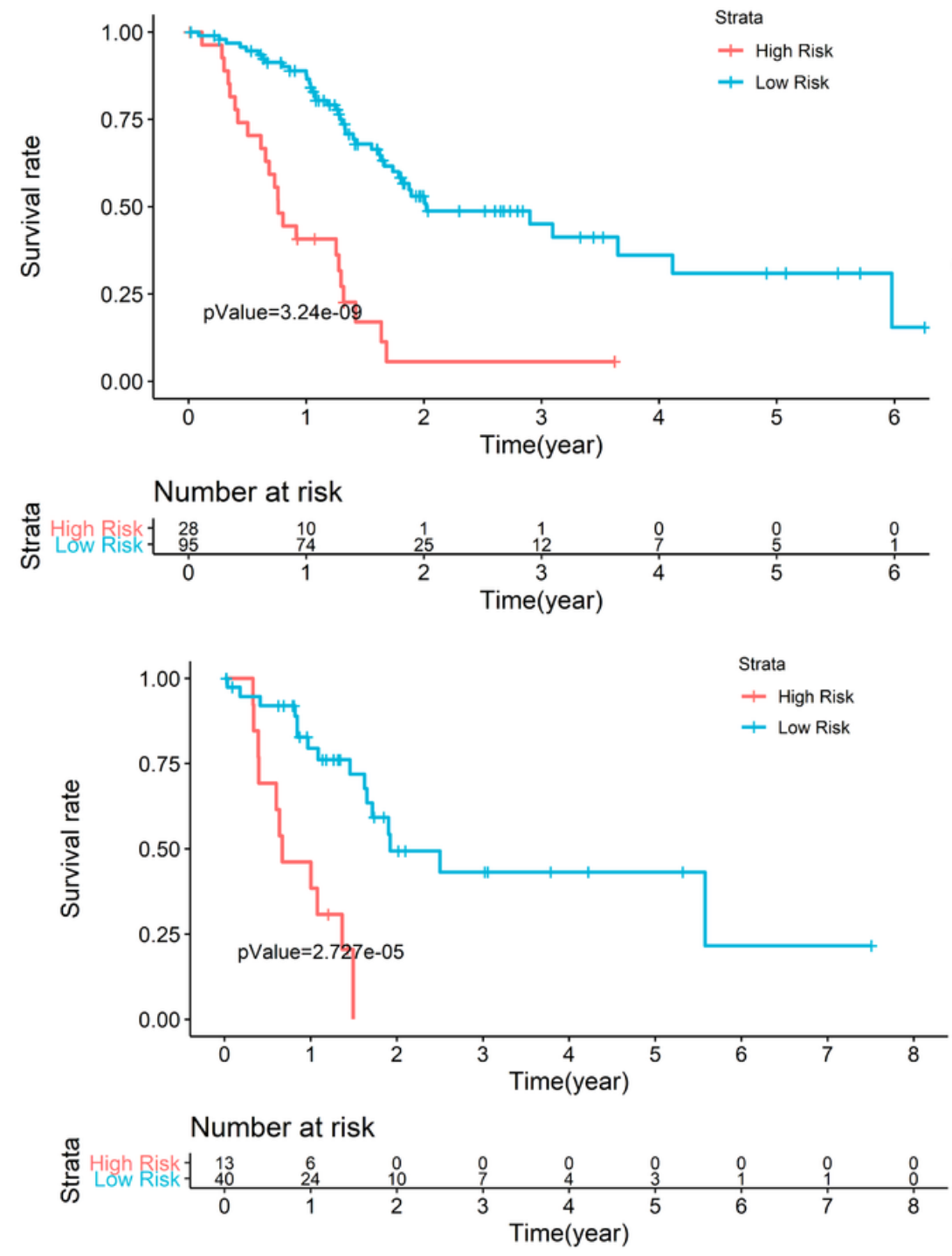

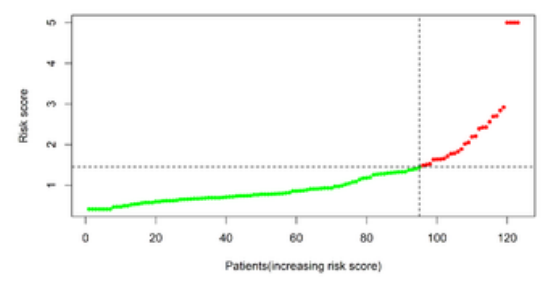

(B)

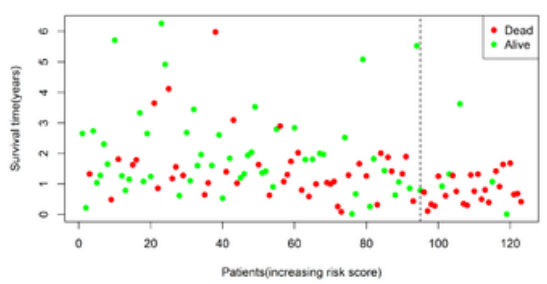

(C)

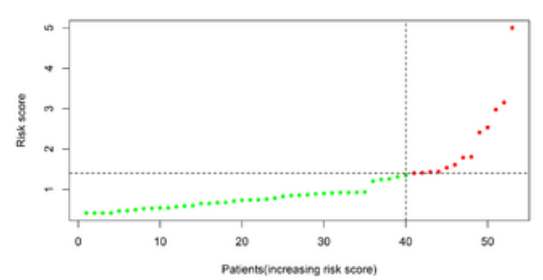

(E)

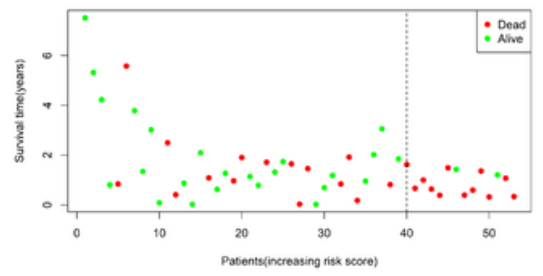

\section{Figure 3}

Clinical evaluation of the risk model in the training and test sets. (A-C) Kaplan-Meier analysis in the training set. (D-F) Kaplan-Meier analysis in the test set. 


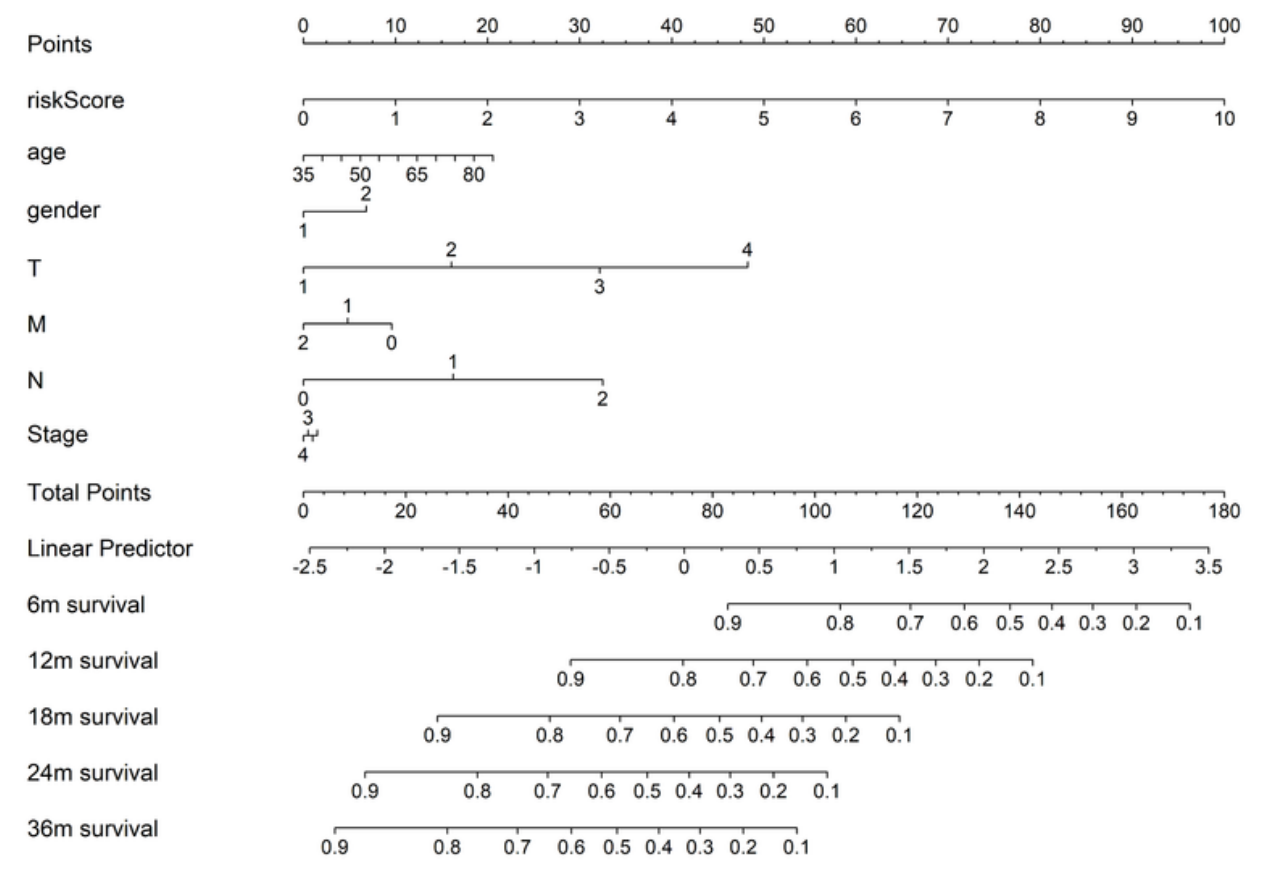

(A)

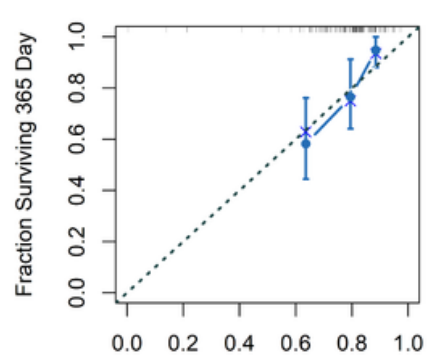

(B)
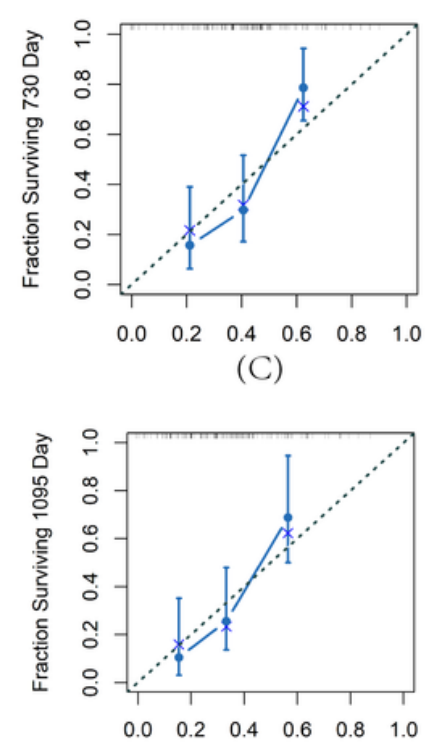

(D)

\section{Figure 4}

The nomogram and calibration plot of the clinical prognostic model. (A) The nomogram of the clinical prognostic model. (B-D) The calibration curves for the 1-, 3- and 5-year survival plots comparing the actual and predicted values. 


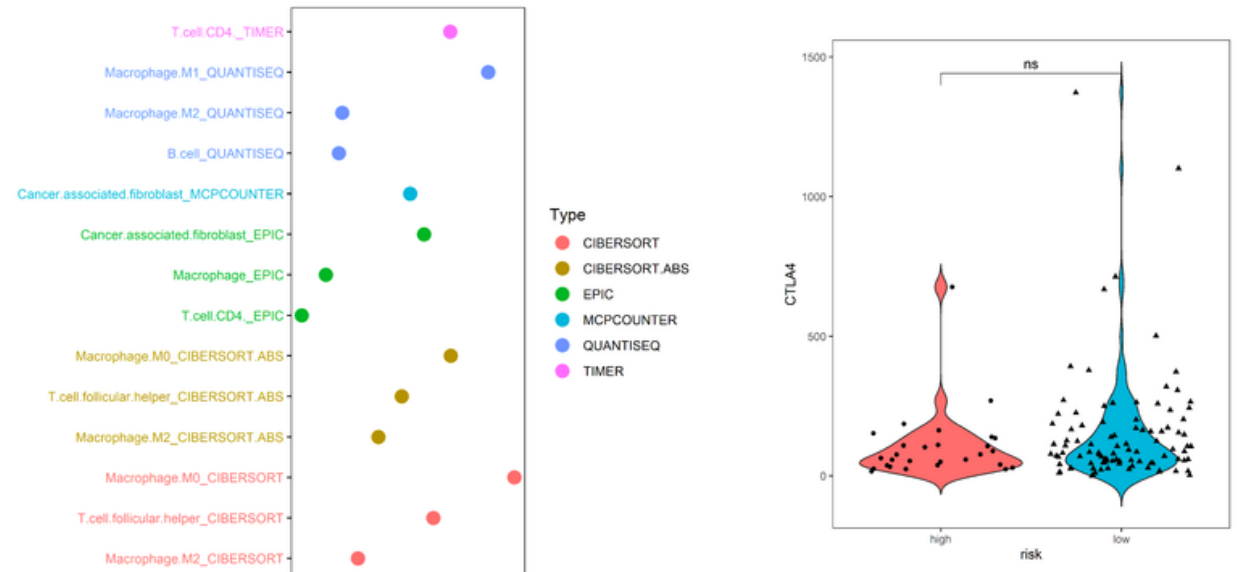

(B)

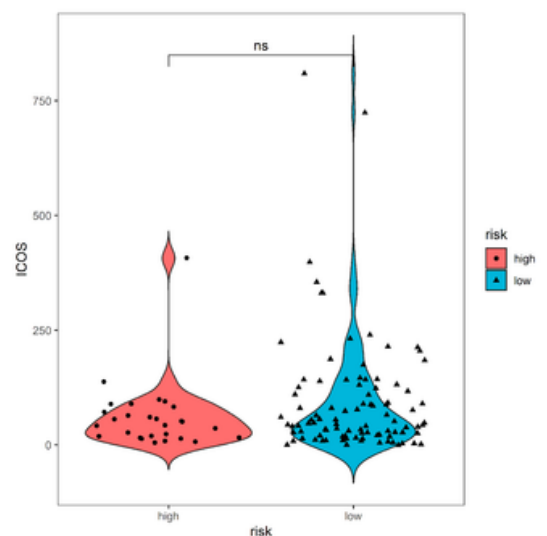

(E)

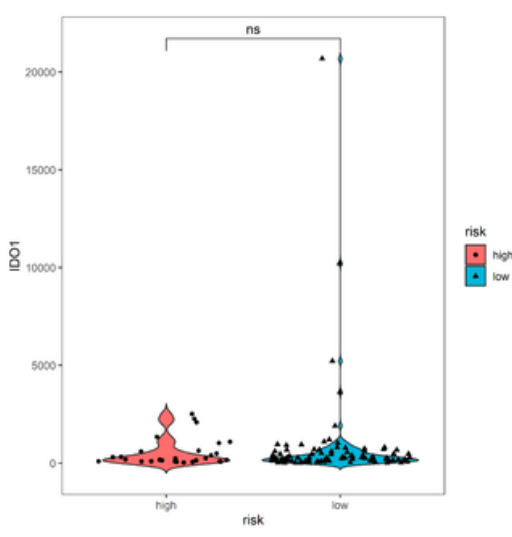

(C)

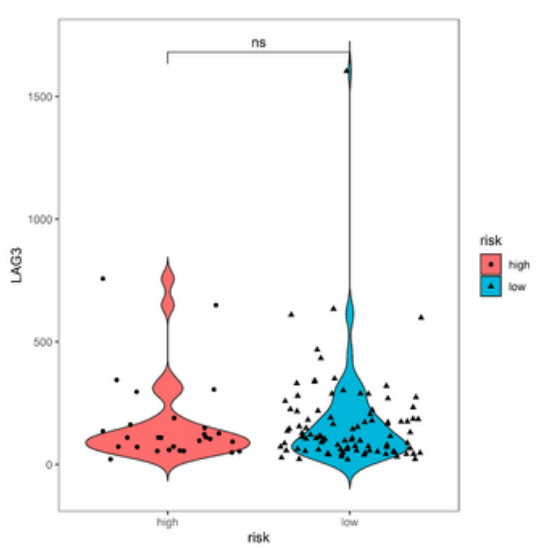

(F)

\section{Figure 5}

The exploration of risk score and immune infiltration status. (A) Lollipop graph of the correlation between immune cell infiltration and risk score. (B-F) Violin plot of risk and ICI targets, including CTLA4, ID01, PDCD1, ICOS, and LAG3.

\section{Supplementary Files}

This is a list of supplementary files associated with this preprint. Click to download.

- SupplementaryTables.xlsx 